\title{
Image based Plant leaf disease detection using Deep learning
}

\section{S. Poornam ${ }^{\text {a. }}$, A. Francis Saviour Devaraj ${ }^{a}$}

${ }^{a}$ Department of Computer Science and Engineering, Kalasalingam Academy of Research and Education, Krishnankoil, Tamil Nadu, India.

* Corresponding Author: its.poornam@gmail.com, francis@klu.ac.in

Received: 11-04-2021, Revised: 11-05-2021, Accepted: 14-05-2021, Published: 30-05-2021

\begin{abstract}
Agriculture is important for India. Every year growing variety of crops is at loss due to inefficiency in shipping, cultivation, pest infestation in crop and storage of government-subsidized crops. There is reduction in production of good crops in both quality and quantity due to Plants being affected by diseases. Hence it is important for early detection and identification of diseases in plants. The proposed methodology consists of collection of Plant leaf dataset, Image preprocessing, Image Augmentation and Neural network training. The dataset is collected from ImageNet for training phase. The $\mathrm{CNN}$ technique is used to differentiate the healthy leaf from disease affected leaf. In image preprocessing resizing the image is carried out to reduce the training phase time. Image augmentation is performed in training phase by applying various transformation function on Plant images. The Network is trained by Caffenet deep learning framework. CNN is trained with ReLu (Rectified Linear Unit). The convolution base of CNN generates features from image through the multiple convolution layers and pooling layers. The classifier part of CNN classifies the image based on the features extracted from the convolution base. The classification is performed through the fully connected layers. The performance is measured using 10-fold cross validation function. The final layer uses activation function like softmax to categorize the outputs.
\end{abstract}

Keywords: Plant disease, Deep learning, CNN, Classification, Artificial Intelligence.

\section{Introduction}

\subsection{Deep learning}

The Deep learning algorithm is used for plant disease recognition and classification problems. The disease identification of plant has become a universal problem. The quality as well as quantity of the agriculture product can be reduced due to plant disease. The quick identification of disease is important for crop growth industry. The main task is to enhance the trait of farm production by the defect identification and disease classification of plant. 
Traditionally with experience or training the humans carried out the crop inspection. In crop protection system early identification of plant disease and accurate result is important. Deep learning approach presents to detect the plant disease and classify the type of disease. Using continuous image capturing the autonomous agriculture vehicles accurately locate psychopathological problem in large cultivation field. The type of disease and disease harshness in plants can be identified by feature extraction and machine learning. The success of machine learning is train an algorithm by access the large amount of data and graphics processing unit (GPU) provides high computation power to achieve the parallelism in data computing.

For pest management early detection of disease is essential strategy. The environment can be polluted by the excess amount of chemical applied and also affects the health of workers in agriculture field and consumer of food. For better crop yield pest management is essential one. To reduce the wastage of applied chemicals, uniform spraying method used that improve the disease control cost. The continuous monitoring of field and quick detection and identification of plant disease are important.

\section{Background}

Here, we present a brief discussion about the plant disease detection and classification that has been implemented by Deep learning algorithms, which can be categorized as; 1 . Dataset collection 2. Image preprocess 3.Image Augmentation 4.Training phase 5.CNN convolution base 6.CNN classifiers 7.Testing Phase.

\section{Literature Review}

Here by we represent the survey on Machine learning based detection using deep learning method of plant disease and classification of various plant disease

Ahmed Khattaba et.al. [1] described to maintain the crop growing environment the early prediction of disease can be carried out which leads to disease outbreak through IOT based precision agriculture monitoring system Information and environmental condition. And internet enabled devices gives the environmental information to the user about the planted crops. To improve the decision making ability of a human the system developed prediction algorithm and artificial intelligence to send the warning messages to user when the plant disease occur. The software module can perform(i)The collected information can be processed(ii) the agriculture module provide the disease models.(ii) Based upon the disease model the amount of pesticides can be applied to the affected area of the plant.

Alexander Johannes et.al. [2] developed a system to minimize the yield losses and efficiency increased by the Phytosanitary treatment. Using mobile phones the early detection of disease can be identified by the technicians and farmer. The plant disease can be solved by combination of image processing technique with statistical inference method. The probability of particular disease can be finding out by plant disease identification. 


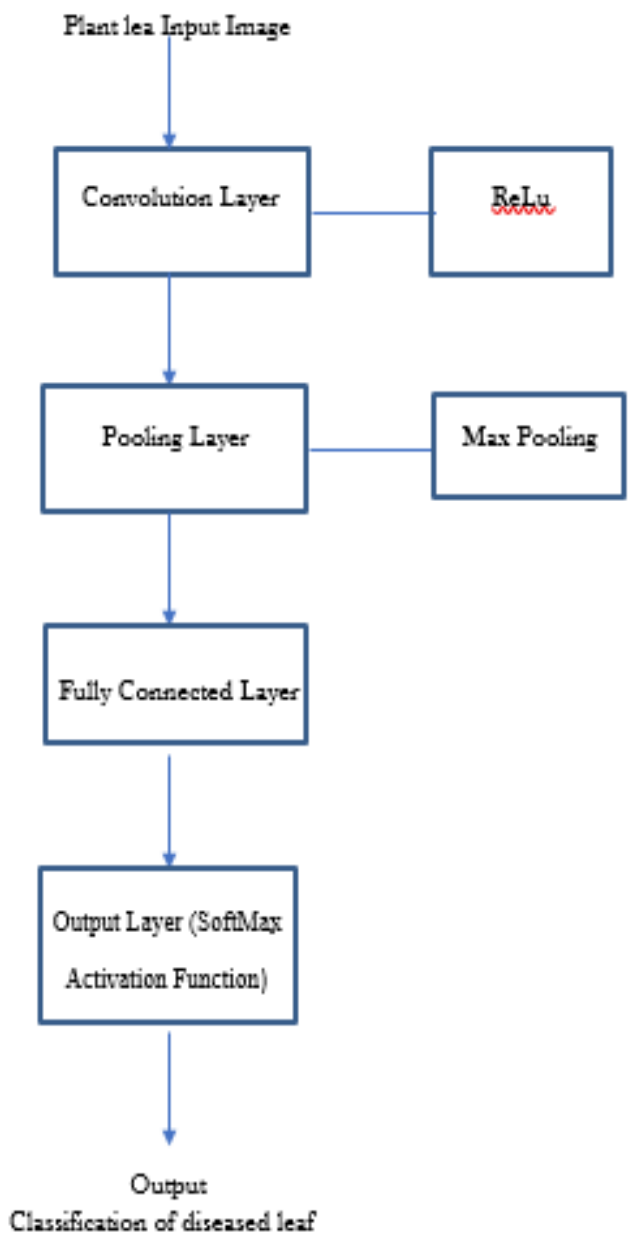

Figure 1. Image Classification technique for leaf disease using CNN.

Kamal K.C et.al. [3] described Continuous monitoring and visual examination of the field by the human sometimes leads to error. It detects the plant disease using depth wise separable convolution based on leaves images. To increase the productivity and improve the plant health based on the characteristics such as color, shape and size.

Srdjan Sladojevic et.al. [4] developed a model for leaf image classification, the plant disease recognition using deep convolution network. By distinguish the plant leaves from their surroundings to recognize various types of plant leaf diseases from the developed model. The plant disease detection from leaf images can be automated by deep learning. . The network can be trained by learn the features of leaf images which distinguish one class from others. Starting from the first convolution layer to the fifth convolution layer, the features can be learned like parts of leaves and shapes are displayed to construct the feature map. 
V.K. Tewari et.al. [5] developed a chemical spraying system due to environmental pollution and economic losses the agrochemical application can be used. The precise agrochemical application develops a chemical spraying system which is based on the severity information about crop disease.

Varalakshmi.P [6] identified the disease infected leaves with the help of automated system by processing the leave images captured by camera. For better disease classification the Active contour edge detection can be used to achieve better results. Active contour edge detection combined with multiclass Support vector machines.

Zahid Iqbala et.al. [7] explains automatic detection of citrus plant disease and classification by the image preprocess, image analysis, pattern extraction, pattern selection and classification methods. Otsu thresolding technique on affected image for background removal during segmentation. To improve the accuracy of classification of diseases using support vector machine (SVM).For segmentation k-mean clustering technique is used. Infected plant images can be divided into many clusters. Each cluster has a different set of pixel values.

Jorge Parraga et.al. [8] Machine learning algorithm has been used for image segmentation and classification. Segmentation can be performed by genetic algorithms, artificial neural network and clustering. Classification can be performed by Support vector machines (SVM) and decision trees.

Konstantinos P et.al [9] Machine learning related application achieved plant illness diagnosis. ANN refers large number of processing layers for deep learning. Convoluted neural network worked as a tool in deep learning. The complex process like pattern recognition for large amount of data can be modeled by $\mathrm{CNN}$.

Vimal K et.al. [10] In this paper detect the disease from rice plant. The disease affected leaves and stems images can be taken from the rice field. For feature extraction the pretrained deep convolution $(\mathrm{SVM})$ network $(\mathrm{CNN})$ has been used and the classification of disease use Support vector Machine. The disease affected rice plant given as an input to the every layers of CNN. The features can be obtained from the final layer of CNN are given to disease classification.

Artzai Picona et.al. [11] combines categorical Meta data (like weather data, location of field and crop identification within convolution neural network. The total number of disease can be obtained from the network final layer.Haseeb Nazkia et.al. [12] improve the efficiency of plant illness detection affects class imbalance in training data. The system needs additional data augmentation measure for balancing the classes of the system.

Jayme G.A.et.al. [13] developed CNN using neural network toolbox for transfer learning data. CNN model trained with three different levels. For training the data $80 \%$ of sample data were used and for data validation $20 \%$ of data were used. Kamlesh et.al. [14] plant protection can be achieved by hyper spectral imaging. The precise and detailed information about an object 
can be extracted from hyper spectral imaging. D. A. Sheikh et.al [15] describes the image processing technique processes the images which are captured by the camera. The converted image sends to RF module. Through this PC connected to robot. The pesticides can be automatically where it is needed.

Vijay Kumar .V et.al [16] developed a system for medical and agriculture the detection of disease can be efficiently performed by image processing and machine learning. For image segmentation mean shift algorithm can be used. Sharma et.al [17] implement the CNN model for plant disease affected images are trained by two CNN model. The leave images from dataset trained first can be called as F-CNN. Jota Shirahatti [18] describes the image training in CNN. The images used in training F-CNN, which can be trained by second model S-CNN.

Mokhtar, U. et.al [19] find the two types of disease on tomato plant perform binary classification using kernel based function with SVM on 200 RGB images. I. Steinwart and A. Christmann [20] describe about the Support vector machines (SVMs) and artificial neural network are commonly used for detecting plant diseases.

\section{Methodology}

\section{Convolution Neural Network}

CNN have two phases:

\section{i) Convolution base}

The convolution base consists of convolution layer and pooling layer. The features are extracted from the dataset images.

\section{ii) Classifiers}

The classifiers of CNN consist of fully connected layer and classify the images based on extracted features from convolution layer.

\section{A. Collection of Dataset}

The dataset used for object identification and evaluate the performance of recognition algorithm during training Phase. The images of plant leafs collects from Image Net database. Both healthy and diseased sample images are collects. All samples are stored in the Joint Photographic Experts Group format.

Source: Kamal KC, Zhendong Yin,Mingyang Wu, Zhilu Wu "Depthwise Separable convolution architectures for plant disease classification" Computers and Electronics in Agriculture (2019) 104948. 


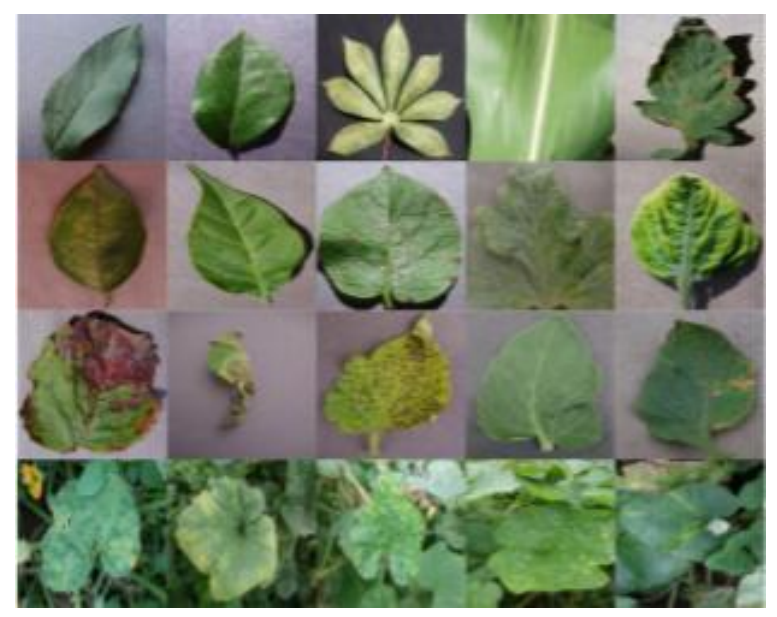

Figure 2. Sample dataset with clean as well as scattered background. [3]

\section{Convolution layer.}

The network is trained to differentiate the plant leaves from surroundings. The first layer of CNN obtains the features from an input leaf image. Using leaf input data to maintain the association among pixels by feature information of image. It holds image matrix and kernel as two inputs. An image in convolution layer executes the process like blur with different filters and edge detection. After the convolution layer the hidden layers generate the feature map. It shares the same weight and bias. The weights allow to be modifies during network training. The weights can be assigned based on the pixels by learning image features.

\section{1) Training Phase}

CNN trained by the ImageNet dataset. From the dataset $90 \%$ images for the phase of training and remaining images used for validation and phase of testing.

Image Preprocess - During learning process the image preprocesses with high resolution and it selects the Region of Interest which differentiates the leaf images from its surroundings. The image size changed to decrease the training time.

Image Augmentation - Image augmentation used for increase the dataset and the network can able to learn more features from image during training phase. The various transformations applied on training dataset to increase the dataset. The affine transformation and perspective transformation applied, this function performs rotations of image on different axis by various degrees.

Pretrained model-The CNN pretrained on Image Net dataset. When pretrained model used the new classifier can be added related to leaf disease classification rather than original classifiers. The weights assigned to all layers .Three categories of pretrained model.

i) Progress the unified model 
Vol. 3 Iss. 1 Year 2021 Poornam S. \& Francis Saviour Devaraj A. /2021

ii) Progress few layers and allow others stable.

iii) Frost the convolution base.

\section{B.Pooling Layer}

Pooling layers performs down sampling of leaf images. It takes the image from previous convolution layer and reduce the extension of each feature map still information about the disease affected area of leaf is maintained. From the feature map the Max pooling takes the largest element for down sampling.

\section{Fully Connected layer}

The image from the pooling layer is in the form of matrix, smoothes the matrix into vector and inserts it into a fully connected layer. The vector generated from the feature map matrix. A network model construct by linking all features together. Finally using activation function used to organize the outputs as diseased leaf, healthy leaf and the type of disease.

\begin{tabular}{|c|c|c|c|}
\hline $\begin{array}{l}\text { Input } \\
\text { Image }\end{array}$ & Original Image & Segmented Image & Classification of disease \\
\hline $\begin{array}{l}\text { Beans } \\
\text { Leaf }\end{array}$ & $\left(\frac{3}{28}\right)$ & & 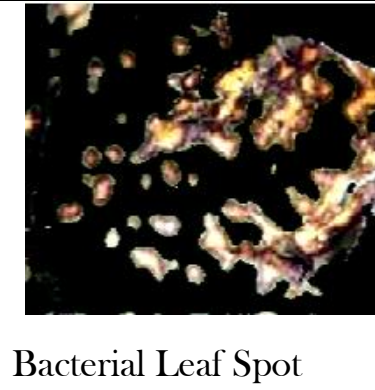 \\
\hline $\begin{array}{l}\text { Rose } \\
\text { Leaf }\end{array}$ & & & $b_{3}$ \\
\hline $\begin{array}{l}\text { Lemo } \\
\text { n Leaf }\end{array}$ & & & 1 \\
\hline
\end{tabular}




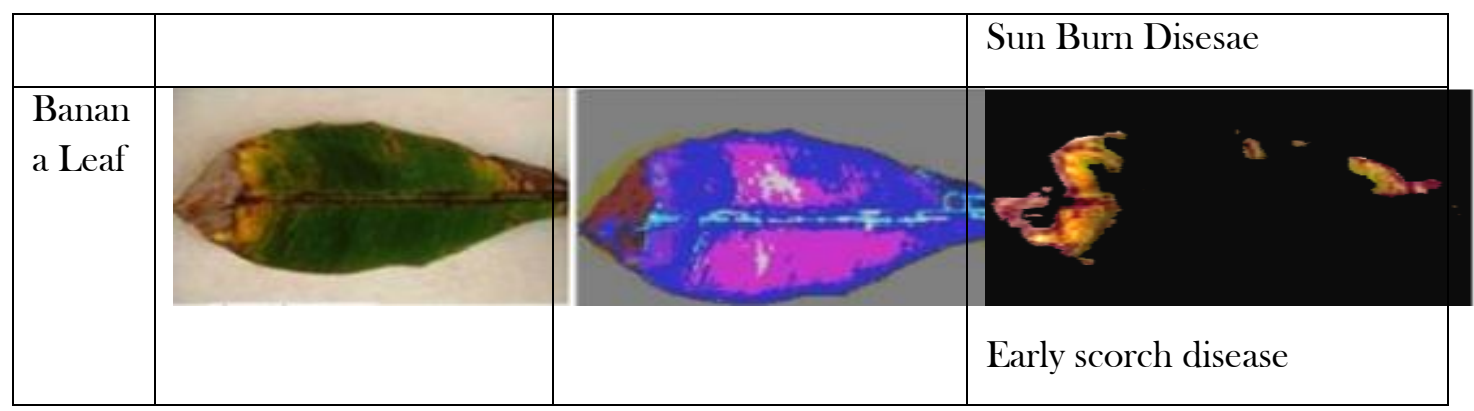

Figure 3. Different types of leaf with segmented images and disease classification images.

\section{2) Testing Phase}

During testing phase, the testing sample input image is given to the convolution layer. Convolution layer processes the image. In convolution layer, convolution is performed on the image and ReLU activation is applied to the matrix. The tested sample leaf images are convoluted with trained sample using its feature map.

The output of many convolution layers is convoluted and the final convolution layer output is fed into pooling layer. The pooling layer performs pooling to decrease dimensionality size. The output of pooling layer acts as an input to a fully connected layer. It flattens the leaf images fed into output layer.

\section{Output Layer}

The output layer uses sigmoid activation function to classify the leaf images into healthy and unhealthy leaf. The sigmoid function is applied for two classification problems and hence we suggest softmax activation function.

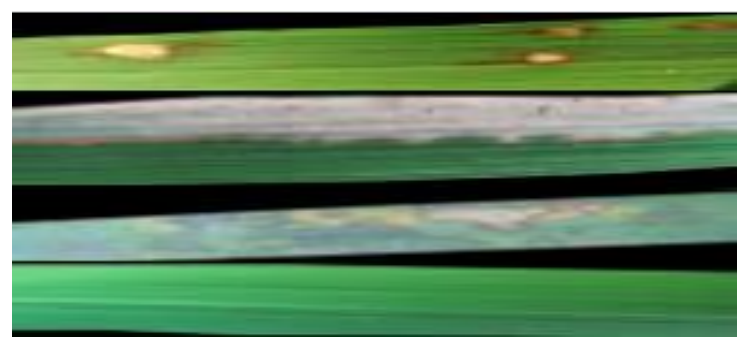

Figure 4. Sample leaf images from dataset with different types of disease. [10]

\begin{tabular}{|c|c|}
\hline $\begin{array}{c}\text { Training-Testing } \\
\text { Division (\%) }\end{array}$ & $\begin{array}{c}\text { Classification } \\
\text { Accuracy (\%) }\end{array}$ \\
\hline $60-40$ & 89.45 \\
$70-30$ & 90.39 \\
$80-20$ & 91.37 \\
\hline
\end{tabular}

Figure 5. Classification accuracy for various training and testing data ratio [10] 
Vol. 3 Iss. 1 Year 2021 Poornam S. \& Francis Saviour Devaraj A. /2021

The softmax activation function applied on leaf images helps to classify the different types of diseases

Source: Vimal K. Shrivastava1, Monoj K. ,Pradhan, Sonajharia Minz, Mahesh P.Thakur” Rice Plant Disease Classification Using Transfer Learning Of Deep Convolution Neural Network” The International Archives of the Photogrammetry,Volume XLII-3/W6, 2019

\begin{tabular}{|c|c|c|c|c|}
\hline \multirow{2}{*}{$\begin{array}{l}\text { Disease } \\
\text { samples }\end{array}$} & \multirow{2}{*}{$\begin{array}{c}\text { No.of images } \\
\text { used for } \\
\text { training }\end{array}$} & \multirow{2}{*}{$\begin{array}{l}\text { No.of images } \\
\text { used for testing }\end{array}$} & \multicolumn{2}{|c|}{ Detection Accuracy } \\
\hline & & & $\begin{array}{c}\text { Classification using } \\
\mathrm{k} \text { means }\end{array}$ & $\begin{array}{l}\text { SVM with } \\
\text { CNN }\end{array}$ \\
\hline Banana & 15 & 10 & 80.00 & 90.00 \\
\hline Beans & 15 & 14 & 92.85 & 92.85 \\
\hline Lemon & 15 & 10 & 90.00 & 100.00 \\
\hline Rose & 15 & 12 & 83.33 & 100 \\
\hline \multicolumn{3}{|c|}{ Overall Accuracy } & 86.54 & 95.71 \\
\hline
\end{tabular}

Table 1. CATEGORIES OF CNN MODEL, METHODS, DATASET \& RESULTS

\begin{tabular}{|c|c|c|c|c|c|c|}
\hline $\begin{array}{l}\text { S. } \\
\text { No }\end{array}$ & $\begin{array}{c}\text { CNN } \\
\text { Model }\end{array}$ & $\begin{array}{l}\text { Algorithm/ } \\
\text { Methods }\end{array}$ & Dataset & $\begin{array}{c}\text { Training/ } \\
\text { Testing data } \\
\text { division }\end{array}$ & $\begin{array}{c}\text { Evaluation } \\
\text { Metrices }\end{array}$ & $\begin{array}{c}\text { Results } \\
\text { Classification } \\
\text { accuracy(\%) }\end{array}$ \\
\hline 1. & $\begin{array}{l}\text { Modified } \\
\text { Mobile Net } \\
\text { Reduced } \\
\text { Mobile } \\
\text { Net[3] }\end{array}$ & $\begin{array}{l}\text { Stochastic } \\
\text { gradient } \\
\text { Descent(SG } \\
\text { D) for } \\
\text { optimization }\end{array}$ & $\begin{array}{l}\text { Plant } \\
\text { Village }\end{array}$ & $80-20$ & $\begin{array}{l}\text { Dice } \\
\text { score(overall } \\
\text { evaluation) }\end{array}$ & $\begin{array}{l}\text { Modified } \\
\text { Mobile Net- } \\
97.65 \\
\text { Reduced } \\
\text { Mobile Net- } \\
98.34\end{array}$ \\
\hline 2. & $\begin{array}{l}\text { Deep } \\
\text { CNN[4] }\end{array}$ & $\begin{array}{l}\text { Deep CNN } \\
\text { with } \\
\text { ReLU(Rectifi } \\
\text { ed Linear } \\
\text { Unit) }\end{array}$ & $\begin{array}{l}\text { images } \\
\text { collected } \\
\text { for the } \\
\text { dataset } \\
\text { were } \\
\text { downloa } \\
\text { ded from } \\
\text { the } \\
\text { Internet }\end{array}$ & & $\begin{array}{l}\text { area under the } \\
\text { curve (AUC), } \\
\text { precision, recall, } \\
\text { F-score and } \\
\text { specificity }\end{array}$ & 96.3 \\
\hline
\end{tabular}


Vol. 3 Iss. 1 Year 2021 Poornam S. \& Francis Saviour Devaraj A. /2021

\begin{tabular}{|c|c|c|c|c|c|c|}
\hline 3. & $\begin{array}{l}\text { AlexNet } \\
{[10]}\end{array}$ & $\begin{array}{l}\text { SVM for } \\
\text { Classification }\end{array}$ & $\begin{array}{l}\text { Indira } \\
\text { Gandhi } \\
\text { Agricultu } \\
\text { ral } \\
\text { Universit } \\
\text { y, Raipur, } \\
\text { Chhattisg } \\
\text { arh, India }\end{array}$ & $80-20$ & $\begin{array}{l}\text { Precision,recall, } \\
\text { misclassification } \\
\text { rate }\end{array}$ & 91.37 \\
\hline 4. & ResNet[11] & $\begin{array}{l}\text { Stocahstic } \\
\text { gradient } \\
\text { descent for } \\
\text { optimization }\end{array}$ & Online & $80-20$ & $\begin{array}{l}\text { Accuracy,sensiti } \\
\text { vity,specificity }\end{array}$ & 98.32 \\
\hline 5. & $\begin{array}{l}\text { F-CNN for } \\
\text { full training } \\
\text { images,S- } \\
\text { CNN for } \\
\text { segmented } \\
\text { training } \\
\text { images[17] }\end{array}$ & - & $\begin{array}{l}\text { Plant } \\
\text { Village }\end{array}$ & $90-10$ & $\begin{array}{l}\text { Dice,similarity,s } \\
\text { ensitivity and } \\
\text { PPV }\end{array}$ & 90.7 \\
\hline
\end{tabular}

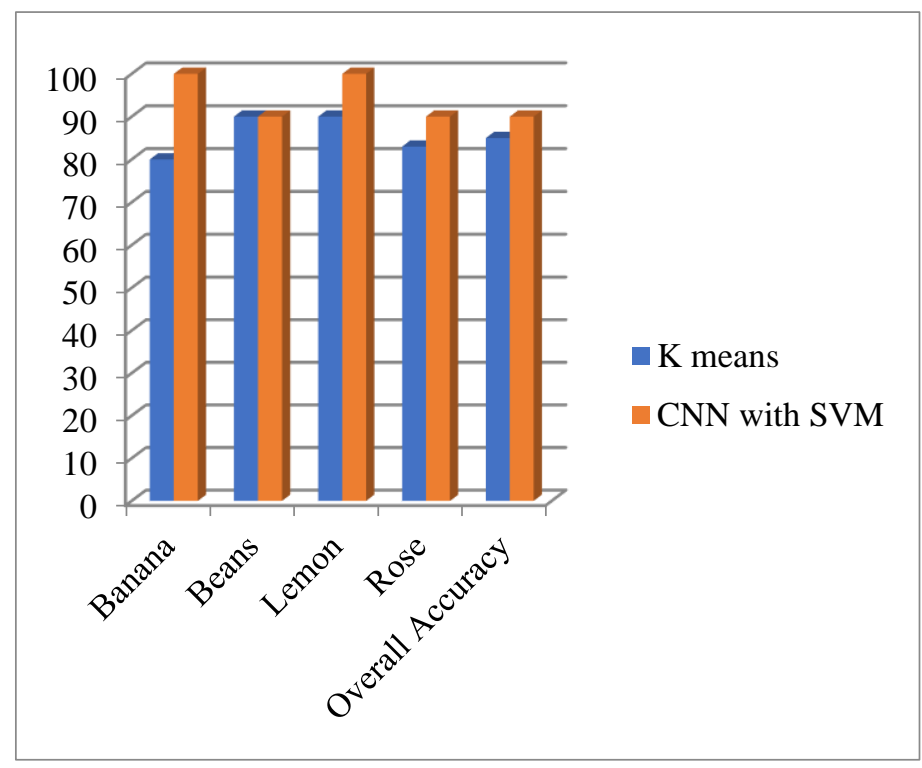

Figure 6. Comparison of Results (Accuracy in \%) 
Vol. 3 Iss. 1 Year 2021 Poornam S. \& Francis Saviour Devaraj A. /2021

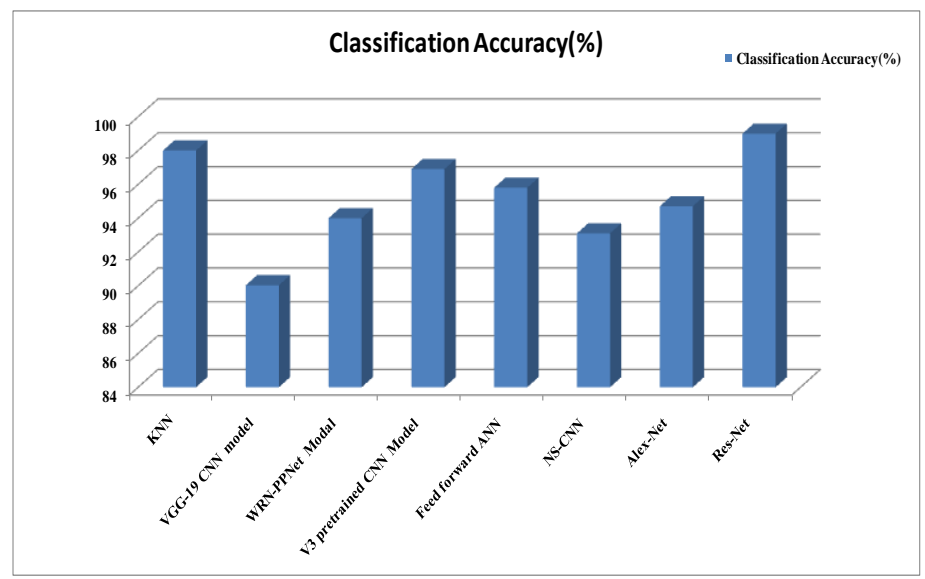

Figure 7. Comparison of accuracy with various $\mathrm{CNN}$ model/Classifiers

\section{Major Findings Ans Suggestions}

Our objective is to detect the leaf disease and classification of disease. The review suggests the CNN will be used to detect the disease type which is learned by the training phase. From the literature survey we find the $\mathrm{CNN}$ is efficient method for disease classification.

\section{Conclusion}

The purpose of applying Deep learning is to improve the work of disease detection and classification. The Network is training by Caffenet deep learning framework. CNN is trained with ReLu. The pretrained model of CNN used transfer learning. The convolution base of CNN generates features from image through the convolution and pooling layers. The classifier part of CNN classifies the image based on the features extracted from the convolution base. The CNN will be used to acquire successive output, identification of disease and type of disease. The pretrained model of $\mathrm{CNN}$ adds the new classifier based upon the purposes we needed. The $80 \%$ of dataset will be used for training phase during pretrained model. The remaining $20 \%$ Of dataset will be used for test phase. So the network can be trained for various applications. The deep learning technique with CNN for the classification of plant illness and predict accurate results.

\section{References}

[1] Khattab, A., Habib, S. E., Ismail, H., Zayan, S., Fahmy, Y., \& Khairy, M. M. (2019). An IoT-based cognitive monitoring system for early plant disease forecast. Computers and Electronics in Agriculture, 166, 105028. https://doi.org/10.1016/j.compag.2019.105028

[2] Johannes, A., Picon, A., Alvarez-Gila, A., Echazarra, J., Rodriguez-Vaamonde, S., Navajas, A. D., \& Ortiz-Barredo, A. (2017). Automatic plant disease diagnosis using mobile capture devices, applied on a wheat use case. Computers and electronics in agriculture, 138, 200-209. https://doi.org/10.1016/j.compag.2017.04.013 
Vol. 3 Iss. 1 Year 2021 Poornam S. \& Francis Saviour Devaraj A. /2021

[3] Kamal, K. C., Yin, Z., Wu, M., \& Wu, Z. (2019). Depthwise separable convolution architectures for plant disease classification. Computers and Electronics in Agriculture, 165, 104948. https://doi.org/10.1016/j.compag.2019.104948

[4] Sladojevic, S., Arsenovic, M., Anderla, A., Culibrk, D., \& Stefanovic, D. (2016). Deep neural networks based recognition of plant diseases by leaf image classification. Computational intelligence and neuroscience.

[5] Tewari, V. K., Pareek, C. M., Lal, G., Dhruw, L. K., \& Singh, N. (2020). Image processing based real-time variable-rate chemical spraying system for disease control in paddy crop. Artificial Intelligence in Agriculture, 4, 21-30. https://doi.org/10.1016/j.aiia.2020.01.002

[6] Varalakshmi, P., \& Aravindkumar, S. (2019). Plant disorder precognition by image based pattern recognition. Procedia Computer Science, 165, 502-510.

[7] Iqbal, Z., Khan, M. A., Sharif, M., Shah, J. H., ur Rehman, M. H., \& Javed, K. (2018). An automated detection and classification of citrus plant diseases using image processing techniques: A review. Computers and electronics in agriculture, 153, 12-32.

[8] Parraga-Alava, J., Cusme, K., Loor, A., \& Santander, E. (2019). RoCoLe: A robusta coffee leaf images dataset for evaluation of machine learning based methods in plant diseases recognition. Data in $\quad$ brief, 25, 104414. https://dx.doi.org/10.1016\%2Fj.dib.2019.104414

[9] Ferentinos, K. P. (2018). Deep learning models for plant disease detection and diagnosis. Computers and Electronics in Agriculture, 145, 311-318. https://doi.org/10.1016/j.compag.2018.01.009

[10] Shrivastava, V. K., Pradhan, M. K., Minz, S., \& Thakur, M. P. (2019). Rice Plant Disease Classification Using Transfer Learning of Deep Convolution Neural Network. International Archives of the Photogrammetry, Remote Sensing \& Spatial Information Sciences, 18-20. http://dx.doi.org/10.1007/s42161-020-00683-3

[11] Picon, A., Seitz, M., Alvarez-Gila, A., Mohnke, P., Ortiz-Barredo, A., \& Echazarra, J. (2019). Crop conditional Convolutional Neural Networks for massive multi-crop plant disease classification over cell phone acquired images taken on real field conditions. Computers and Electronics in Agriculture, 167, 105093. https://doi.org/10.1016/j.compag.2019.105093

[12] Nazki, H., Yoon, S., Fuentes, A., \& Park, D. S. (2020). Unsupervised image translation using adversarial networks for improved plant disease recognition. Computers and Electronics in Agriculture, 168, 105117. https://doi.org/10.1016/j.compag.2019.105117

[13] Barbedo, J. G. (2018). Factors influencing the use of deep learning for plant disease recognition. Biosystems engineering, 172, 84-91.

[14] Golhani, K., Balasundram, S. K., Vadamalai, G., \& Pradhan, B. (2018). A review of neural networks in plant disease detection using hyperspectral data. Information Processing in Agriculture, 5(3), 354-371. https://doi.org/10.1016/j.inpa.2018.05.002 
Vol. 3 Iss. 1 Year 2021 Poornam S. \& Francis Saviour Devaraj A. /2021

[15] Shaikh, D. A., Ghorale Akshay, G., Chaudhari Prashant, A., Kale Parmeshwar, L., (2016). Intelligent Autonomous Farming Robot with Plant Disease Detection using Image Processing. International Journal of Advanced Research in Computer and Communication Engineering, 5. https://doi.org/10.17148/IJARCCE.2016.54248

[16] Vijay Kumar, V., Vani, K.S., Acharya, (2018). Agricultural Robot: Leaf Disease Detection and Monitoring the Field Condition Using Machine Learning and Image Processing, International Journal of Computational Intelligence Research, 14 (7) 551561.

[17] P. Sharma, Y. Paul Singh Berwal, W. Ghai, Performance Analysis of Deep Learning CNN Models for Disease Detection in Plants using Image Segmentation, Information Processing in Agriculture (2019).

[18] Shirahatti, J., Patil, R., \& Akulwar, P. (2018). A survey paper on plant disease identification using machine learning approach. In 2018 3rd International Conference on Communication and Electronics Systems (ICCES), IEEE. 1171-1174.

[19] Mokhtar, U., Ali, M. A., Hassanien, A. E., \& Hefny, H. (2015). Identifying two of tomatoes leaf viruses using support vector machine. In Information Systems Design and Intelligent Applications, Springer, New Delhi. 771-782.

[20] Steinwart, I., Christmann, A., (2008). Support Vector Machines, Springer Science \& Business Media, New York, NY, USA.

\section{Acknowledgements}

The authors declare that they have no conflict of interest.

\section{Conflict of interest}

The authors declare that they have no conflict of interest.

\section{About The License}

(C) 2021 The Authors. This work is licensed under a Creative Commons Attribution 4.0 International License which permits unrestricted use, provided the original author and source are credited. 